\title{
Neuerungen bei der Schweizerischen Gesellschaft für Notfall- und Rettungsmedizin SGNOR
}

\author{
L. Bernoulli, D. Scharplatz ${ }^{b}$
}

La version française suivra

\footnotetext{
a Präsident SGNOR

b Leiter Arbeitsgruppe Integration Klinische Notfallmedizin SGNOR
}

Korrespondenz:

Dr. med. Lion Bernoulli

Universitätsspital

Institut für Anästhesiologie

Rämistrasse 100

CH-8091 Zürich

E-Mail: lion.bernoulli@usz.ch
Die Schweizerische Gesellschaft für Notfall- und Rettungsmedizin SGNOR hat vor Jahresfrist beschlossen, sich in Richtung Klinische Notfallmedizin und Katastrophenmedizin zu öffnen. Dazu mussten einige Umstrukturierungen vorgenommen werden, insbesondere eine Statutenrevision, welche von der Mitgliederversammlung am 22. April 2005 angenommen wurde.

Mit der FMH wurden Kontakte bezüglich Schaffung eines Fähigkeitsausweises Klinische Notfallmedizin aufgenommen. Als ersten Schritt hat die SGNOR ein «Programm Klinische Notfallmedizin» erarbeitet, welches diesem Text folgt. Das Programm richtet sich an Ärzte, welche in leitender Funktion auf einer Notfallstation tätig und Fachärzte für Allgemeinmedizin, Anästhesiologie, Chirurgie, Orthopädische Chirurgie und Traumatologie des Bewegungsapparates, Innere Medizin, Pädiatrie, Kinderchirurgie oder Intensivmedizin sind. Gemeinsam mit ihnen und der FMH will die SGNOR einen Fähigkeitsausweis Klinische Notfallmedizin ausarbeiten. Deshalb können Inhaber einer durch die SGNOR ausgestellten Bestätigung des erfüllten Programms Klinische Notfallmedizin nun auch als ordentliche Mitglieder in die SGNOR aufgenommen werden.
Fachärzte, welche bereits über die Anforderungen im «Programm Klinische Notfallmedizin» verfügen, sind deshalb gebeten, sich bei der Sekretärin der SGNOR, Doris Oechslin, zu melden, um die notwendigen Unterlagen zu erhalten.

Die SGNOR ist überzeugt, mit diesem Beschluss einen wichtigen Schritt über die Spitalschwelle gemacht zu haben, darf doch die strukturierte Weiterbildung in Notfallmedizin nicht vor der Spitalpforte haltmachen; sie muss auch die Behandlung auf der Notfallstation nach gleichen Kriterien und Grundsätzen garantieren. Nur so bleibt die fachliche Kohärenz bestehen und nur so können Dissonanzen bei der Übergabe der Patienten auf der Notfallstation vermieden werden. Die SGNOR dankt Ihnen allen für Ihre Mithilfe und freut sich auf eine erspriessliche Zusammenarbeit.

Ferner wurde ein Curriculum «Leitender Notarzt» ausgearbeitet, dessen Weiterbildung gemeinsam mit dem CEFOCA (Centre de formation en médicine militaire et de catastrophe) erfolgt. Die Kursabsolventen CEFOCA sind nach dieser Ausbildung Leitende Notärzte SGNOR; hier bedarf es keiner neuen Regelung. 


\title{
Programm Klinische Notfallmedizin SGNOR
}

\author{
L. Bernoulli ${ }^{a}$ L. Anselmi ${ }^{b}$
}

La version française suivra

\section{Ziel und Zweck}

Das Programm Klinische Notfallmedizin SGNOR definiert die Kriterien, über welche Ärzte* verfügen sollten, die in leitender Funktion auf einer Notfallstation tätig sind. Ziel ist die Einführung eines eigenständigen Fachbereichs Klinische Notfallmedizin. Im Sinne einer vorläufigen Regelung dient das Programm als Basis zur Erlangung des zu schaffenden Fähigkeitsausweises Klinische Notfallmedizin.

\section{Voraussetzungen}

Eidgenössischer oder anerkannter ausländischer Facharzttitel für Allgemeinmedizin, Anästhesiologie, Chirurgie, Orthopädische Chirurgie und Traumatologie des Bewegungsapparates, Innere Medizin, Pädiatrie, Kinderchirurgie oder Intensivmedizin.

\section{Inhalt}

3.1 Erfolgreich bestandene Kurse in:

- Advanced Cardiovascular Life Support nach American Heart Association (ACLS-AHA);

a Präsident SGNOR

b Vizepräsident SGNOR

* Dieses Programm gilt in gleichem Masse für Ärztinnen und Ärzte. Zur besseren Lesbarkeit werden im Text nur männliche Personenbezeichnungen verwendet. Wir bitten die Leserinnen um Verständnis.

\section{Korrespondenz:}

SGNOR

Postfach 1154

CH-8032 Zürich

Tel. 0447991546

Fax 0447991547

E-Mail sgnor.ssmus@bluewin.ch

Internet:

www.sgnor.ch, www.ssmus.ch
- $\quad$ Advanced Trauma Life Support ${ }^{\circledR}\left(\right.$ ATLS $\left.^{\circledR}\right)$;

- Pediatric Advanced Life Support nach American Heart Association (PALS-AHA);

- gleichwertige Kurse können von der SGNOR anerkannt werden.

3.2 Nach Erlangung eines eidgenössischen oder anerkannten ausländischen Facharzttitels 100\%-Tätigkeit für 1 Jahr oder 50\%-Tätigkeit für 2 Jahre auf einer Notfallstation, die für eine Facharztausbildung anerkannt ist.

\section{4. Übergangsbestimmungen}

Für 5 Jahre nach Inkrafttreten des Programmes gelten folgende Mindestanforderungen:

4.1 Eidgenössischer oder anerkannter ausländischer Facharzttitel für Allgemeinmedizin,
Anästhesiologie, Chirurgie, Orthopädische Chirurgie und Traumatologie des Bewegungsapparates, Innere Medizin, Pädiatrie, Kinderchirurgie oder Intensivmedizin.

4.2 Aktuelle Tätigkeit als Leiter einer Notfallstation oder regelmässige Tätigkeit auf einer Notfallstation im Rahmen einer beteiligten Fachdisziplin während mindestens 2 Jahren.

4.3 Einer der erfolgreich bestandenen Kurse in:

- Advanced Cardiovascular Life Support nach American Heart Association (ACLS-AHA);

- Advanced Trauma Life Support ${ }^{\circledR}\left(\right.$ ATLS $\left.^{\circledR}\right)$;

- Pediatric Advanced Life Support nach American Heart Association (PALS-AHA);

- gleichwertige Kurse können von der SGNOR anerkannt werden.

\section{Bestätigung}

Die Bestätigung des erfüllten Programms erfolgt durch die Weiter- und Fortbildungskommission.

\section{Gültigkeit und Fortbildung}

6.1 Das erfüllte Programm Klinische Notfallmedizin ist 5 Jahre gültig.

6.2 Die Gültigkeit kann jeweils um 5 Jahre verlängert werden, wenn 50 durch die SGNOR anerkannte Fortbildungsstunden gemäss Fortbildungsordnung Notarzt SGNOR absolviert werden.

6.3 Die Bestätigung der erfüllten Fortbildung erfolgt durch die Weiter- und Fortbildungskommission.

\section{7. Änderungen}

Über Änderungen des Programms entscheidet die Mitgliederversammlung.

Genehmigt von der Mitgliederversammlung am 22. April 2005 in Luzern. 